\title{
Empirical determination of the mechanical properties of Vacuum Packed Particles
}

\author{
Piotr Bartkowski ${ }^{1}{ }^{*}$, Robert Zalewski ${ }^{1}$ \\ ${ }^{1}$ Institute of Machines Design Fundamentals, Warsaw University of Technology, Narbutta 84, 02-254 \\ Warsaw, Poland
}

\begin{abstract}
In this work the experimental test of Vacuum Packed Particles (VPP) under 3-point bending are presented. VPP it is the structure compose of grains inside the plastomer coating. When the pressure inside is equal or higher than zero structure behave like a liquid, otherwise like a elasto-plastic solid. Three types of grain material and different values of underpressure were tested. Results presented in this paper will be used in the further work for the numerical model validation procedure.
\end{abstract}

Keywords: Vacuum Packed Particles, Smart Materials

\section{Introduction}

Due to low density and relatively low production costs the Vacuum Packed Particles (VPP) can be competitive alternative for the traditional smart material including magnetorheological fluid or electreological fluids. Scientists from around the worlds have been investigating the mechanical properties and looking for the engineering applications of VPP for many years. In this field, the application process has significantly exceeded the approach to modeling. The first and most famous application using VPP is the emergency mattress using for transporting the wounded [1]. The another application in medicine is the innovatory support for endoscope that allow to change the bending stiffness of the devise. The authors of paper [2] described the influence of the grain size for the behavior of the whole structure. VPP have also found applications in robotics. The most popular is the universal gripper able to carrying devices with different shapes [3]. The other interesting application in robotics is the ChemBot described in paper [4]. The smart material described in this paper can be also use for the active damping of mechanical vibration or for the crash energy absorbing. The interesting devices is the linear controllably linear damper made of VPP and described in paper [5]. Other interesting devise is the multiaxial impact damper able to absorb the impact energy [6].

The growing popularity of VPP encouraged scientist to testing and modeling of this structure. From the mechanical point of view VPP behave like a elasto-plastic solid with strong hardening. It can be found in literature the plenty of results for the uniaxial test of VPP

\footnotetext{
${ }^{*}$ Corresponding author: bartkowski_piotr@onet.pl

Reviewers: Marcin Kubiak, Milan Sága
} 
$[7,8]$. Scientists are also working on dynamic test of this structure and the results are presented in papers $[9,10]$.

Despite the fact that Vacuum Packed Particles are studied in numerous research centers, many problems are still unsolved. The previously published results pertained only to the behavior of the material in simple uniaxial stress and strain state. There is still lack of material characteristic data under complex stress state. In this paper the result of the 3 point bending are presented. Test for the different under pressure value and grain type were performed. The experiments allow to better understanding of the material behavior under complex stress state.

\section{Method of testing}

The tested beam is shown in Fig. 1 . The dimension of the elements is equal to $60 \times 20 \times 3000$ (Fig. 3). Beam was formed in the dedicated devise to obtain the correct and repeatable dimension (Fig. 3). After proper grain placement the under pressure was set and tests were performed on the testing machine SHIMADZU EZ-LX equipped with force load cell max $5000 \mathrm{kN}$ (Fig. 4) and the special devise for 3-point bending. During the experiments the value of force and displacement were measured. The influence of the under pressure inside the plastic envelope was tested. Experiments were performed for three different grain material what is precisely described in the next chapter.

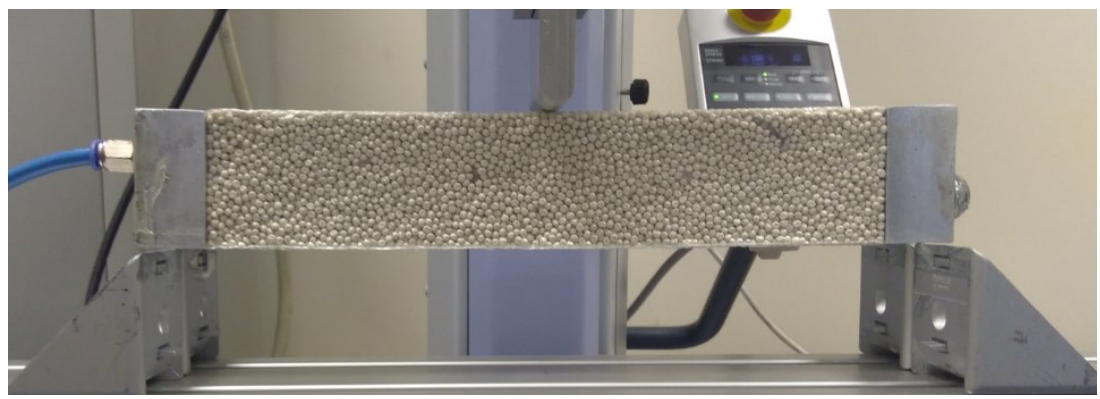

Fig. 1. Tested beam

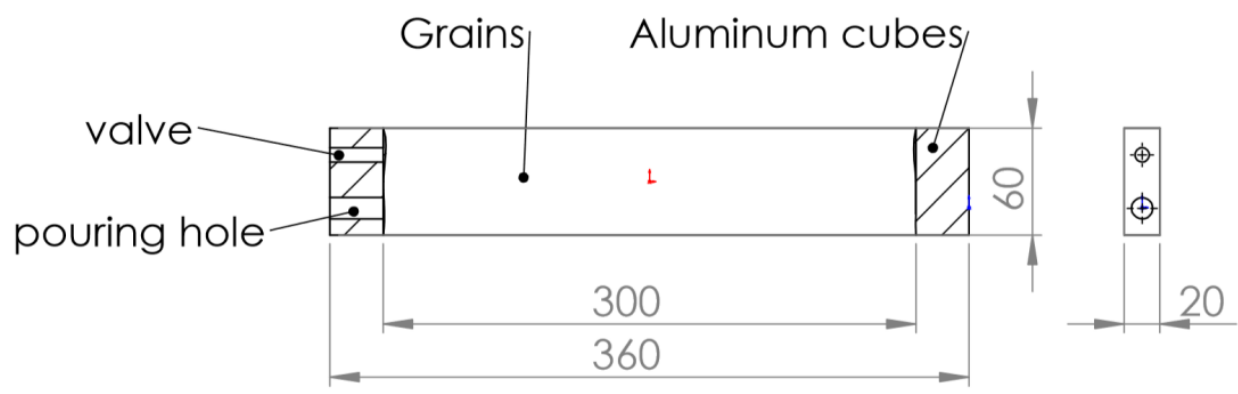

Fig. 2. Dimensions of tested element 


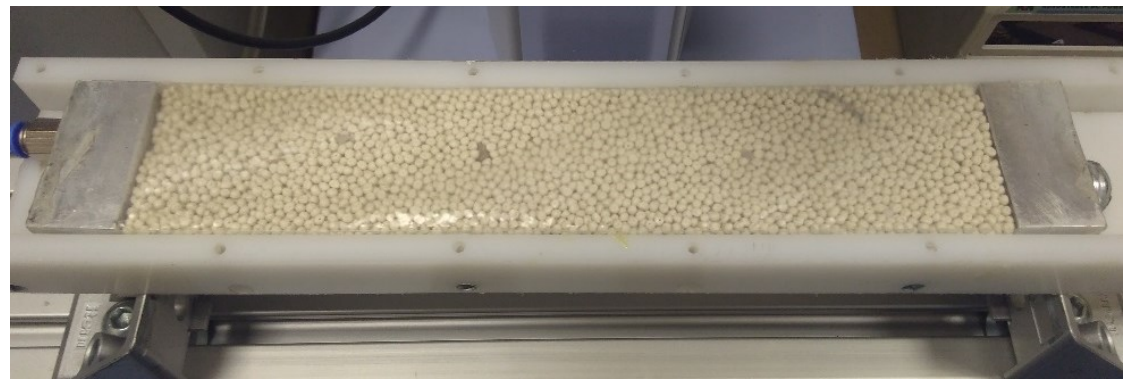

Fig. 3. Form for beam shaping

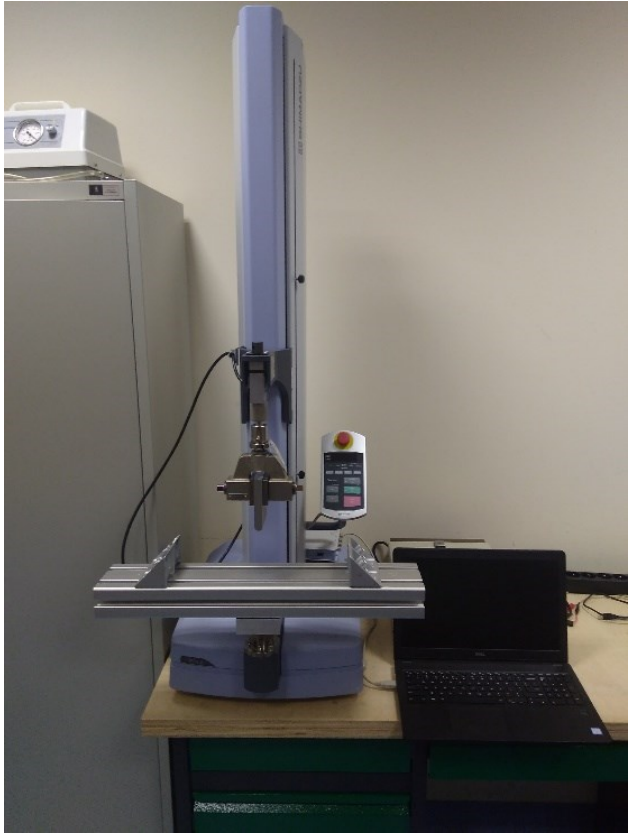

Fig. 4. Testing machine

\section{Types of material}

In the experiments three types of grain material, illustrated on Figure 5 were tested. Grains were ball-shape with diameter $3 \mathrm{~mm}$. The same material fitting was used in each beam, equal to $66 \%$. The mechanical properties of the grain material are presented in table 1 . The VPP coating, made of Polyethylene, was the same in each material sample. The yield curve for this material is presented in Fig. 6 . 


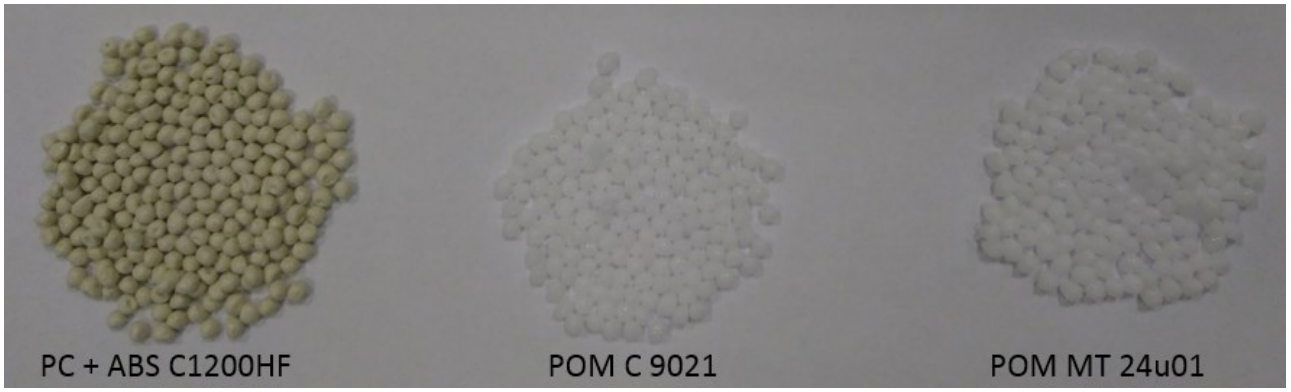

Fig. 5. Dedicated test stand

Table 1. Mechanical properties of the grain materials

\begin{tabular}{|c|c|c|c|}
\hline & $\begin{array}{c}\text { Young modulus } \\
{[\mathrm{MPa}]}\end{array}$ & $\begin{array}{c}\text { Yield stress } \\
{[\mathrm{MPa}]}\end{array}$ & $\begin{array}{c}\text { Nominal strain } \\
\text { at break [\%] }\end{array}$ \\
\hline PC/ABS C12HF & 2360 & 55 & $>50$ \\
\hline POM C 9021 & 2500 & 48 & 16 \\
\hline POM MT24u01 & 2900 & 65 & 17 \\
\hline
\end{tabular}

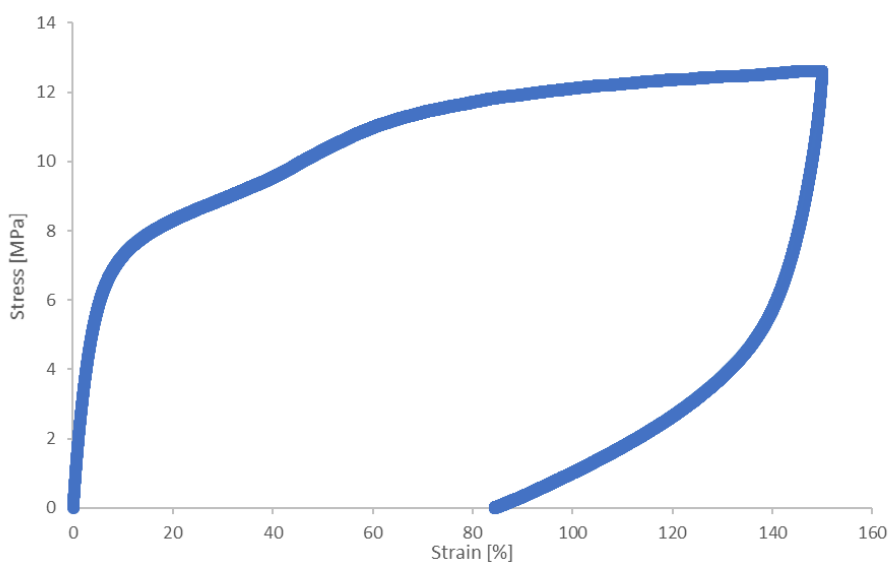

Fig. 6. Yield curve for PE

\section{Results}

In this chapter results of the experiments are presented. Force-displacement curve for each material is shown (Fig. 7-9). During the experiment three samples for each under pressure and material were tested to checked that the results are repeatable (Fig. 13). In the Figure 1012 the basic mechanical properties in function of under pressure are presented. 


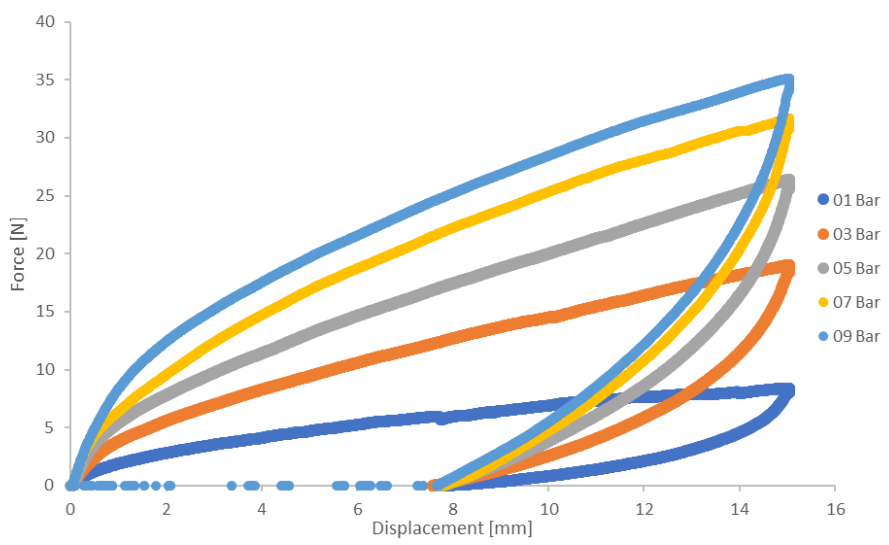

Fig. 7. Force/dispalcement curve for PC/ABS C12HF

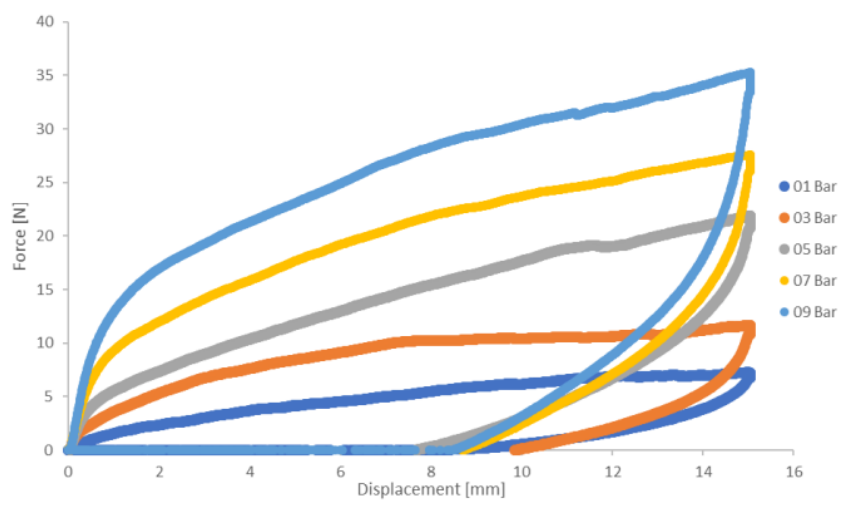

Fig. 8. Force/displacement curve for POM C 9021

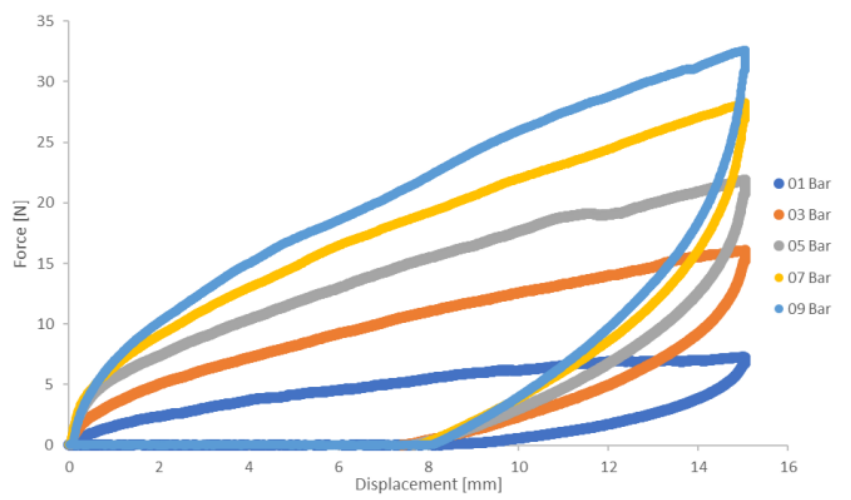

Fig. 9. Force/displacement curve for POM MT24u01 


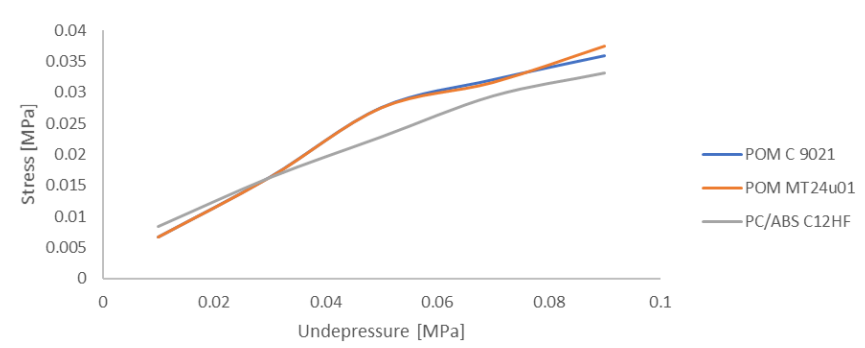

Fig. 10. Stress $(0.2 \%)$

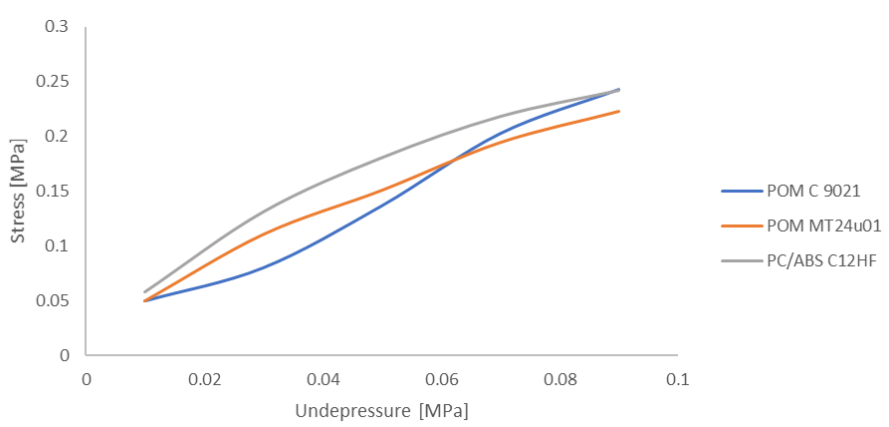

Fig. 11. Max stresses

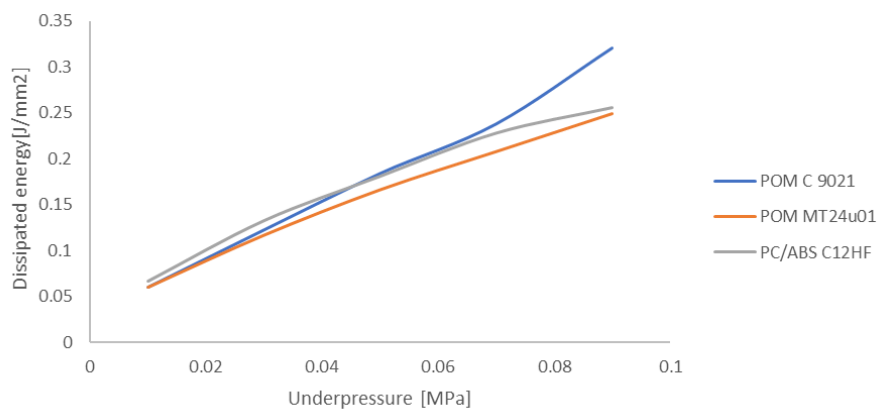

Fig. 12. Dissipated energy

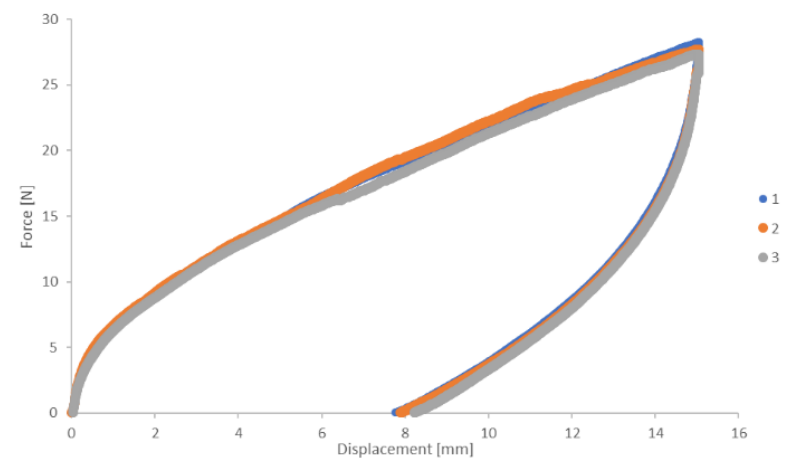

Fig. 13. Test for underpressure 0.7 bar 


\section{Conclusions}

In this work the results of the experimental test of Vacuum Packed Particles are presented. Three-point bending test was performed to achieve the complex stress state. Three types of materials with different underpressure value were tested.

The results show that underpressure inside the envelope affect the mechanical properties of the material (Fig. 10, 11, 12). Beam behave like a elasto-plastic solid with quite large elastic part of the strain what can be cause by plastomer envelope which is stretch during the entire process. Vacuum Packed Particles behave in this system like a composite structure. Noticeable is the lack of significant influence of the grain type on the behavior of the whole structure, what was found in case of uniaxial tension and compression. The results obtained during the experiments will be used in the further work on the modeling of VPP.

This work was carried out within the project no. 2016/23/N/ST8/02056 founded by National Science Centre, Poland.

\section{References}

1. Luscombe, M. and Williams, J. (2003). Comparison of a long spinal board and vacuum mattress for spinal immobilisation. Emergency medicine journal, 20(5):476-478

2. Loeve, A. J., van de Ven, O. S., Vogel, J. G., Breedveld, P., and Dankelman, J. (2010). Vacuum packed particles as flexible endoscope guides with controllable rigidity. Granular matter, 12(6):543-554

3. Brown, E., Rodenberg, N., Amend, J., Mozeika, A., Steltz, E., Zakin, M. R., Lipson, H., and Jaeger, H. M. (2010). Universal robotic gripper based on the jamming of granular material. Proceedings of the National Academy of Sciences, 107(44):18809-18814

4. Annan Mozeika, Erik Steltz, Heinrich M. Jaeger. The first steps of a robot based on jamming skin enabled locomotion, October 2009 IROS'09: Proceedings of the 2009 IEEE/RSJ international conference on Intelligent robots and systems, IEEE Press

5. Zalewski, R., Chodkiewicz, P., and Shillor, M. (2016). Vibrations of a mass-spring system using a granular-material damper. Applied Mathematical Modelling, 40(1718):8033-8047

6. Bartkowski, P. and Zalewski, R. (2018). A concept of smart multiaxial impact damper made of vacuum packed particles. In MATEC Web of Conferences, volume 157, page 05001. EDP Sciences

7. Zalewski, R. and Chodkiewicz, P. (2014). Gubanov model for vacuum packed particles. In Mechatronics 2013, pages 57-63. Springer

8. Zalewski, R., Chodkiewicz, P., and Shillor, M. (2016). Vibrations of a mass-spring system using a granular-material damper. Applied Mathematical Modelling, 40(1718):8033-8047

9. Bajkowski J.M., Dyniewicz B., Bajer C.I., 2015, Damping properties of a beam with vacuum-packed granular damper, Journal of Sound and Vibration, 341, 74-85

10. Szmidt T., Zalewski R., 2014, Inertially excited beam vibrations damped by vacuum packed particles, Smart Materials and Structures, 23, 10 